\title{
Sequential Desynchronization in Networks of Spiking Neurons with Partial Reset
}

\author{
Christoph Kirst, ${ }^{1,3}$ Theo Geisel, ${ }^{1,2,3}$ and Marc Timme ${ }^{1,2}$ \\ ${ }^{1}$ Max Planck Institute for Dynamics and Self-Organization (MPIDS), 37073 Göttingen, Germany \\ ${ }^{2}$ Bernstein Center for Computational Neuroscience (BCCN) Göttingen, 37073 Göttingen, Germany \\ ${ }^{3}$ Faculty of Physics, Georg August University Göttingen, 37077 Göttingen, Germany
}

(Received 11 July 2008; published 9 February 2009)

\begin{abstract}
The response of a neuron to synaptic input strongly depends on whether or not the neuron has just emitted a spike. We propose a neuron model that after spike emission exhibits a partial response to residual input charges and study its collective network dynamics analytically. We uncover a desynchronization mechanism that causes a sequential desynchronization transition: In globally coupled neurons an increase in the strength of the partial response induces a sequence of bifurcations from states with large clusters of synchronously firing neurons, through states with smaller clusters to completely asynchronous spiking. We briefly discuss key consequences of this mechanism for more general networks of biophysical neurons.
\end{abstract}

The brain processes information in networks of neurons, which interact by sending and receiving electrical pulses called action potentials or spikes. The response of a neuron to incoming signals strongly depends on whether or not it has just sent a spike itself. After the initiation of a spike the membrane potential at the cell body (soma) is reset towards some potential and the response to further synaptic input is reduced due to the refractoriness of the neuron [1]. The dendritic part of the neuron where incoming signals are integrated is affected only indirectly by this reset due to intraneuronal interactions [2-4].

To characterize this effect several multicompartment models have been proposed in which different parts of a single neuron interact [2]. For instance, in a twocompartment model [3] of coupled dendrite and soma, the membrane potential at the soma is reset after spike emission while the dendritic dynamics is affected only by the resistive coupling from the soma to the dendrite. This accounts for the fact that in several kinds of neurons residual charge remains on the dendrite (following the somatic reset) that is then transferred to the soma $[4,5]$. Thus the dynamics of the individual neurons is modified which severely affects the collective capabilities of networks of such neurons.

In this Letter we propose a simple neuron model which captures the response to residual input charges following spike emission in the form of a partial reset and at the same time allows an analytical study of the collective network dynamics. A fraction $c \in[0,1]$ of the residual suprathreshold input charge is kept by the neuron after reset. For $c=$ 0 all additional input charge not needed to trigger a spike is lost after spike emission, whereas for $c=1$ the total input charge is conserved [6]. Although the regime $0<c<1$ is likely to be the biologically more relevant, to our knowledge, there are so far no systematic studies of the dynamics of networks of neurons with partial response. To reveal the basic mechanisms underlying the collective dynamics of networks of such neurons we focus on globally and homogeneously coupled neurons. Despite their simplicity these networks already exhibit a rich variety of dynamics that is controlled by the partial reset. In particular we find and show analytically that for a broad class of neurons there is a desynchronization transition in the network dynamics determined by a sequence of bifurcations: For small $c$ the fully synchronous state coexists with a variety of cluster states (cf. [7]), with differently sized groups of synchronously firing neurons. With increasing $c$, states with clusters of size $a$ and larger become sequentially unstable at bifurcation points $c_{\mathrm{cr}}^{(a)}$ satisfying $0 \leq c_{\mathrm{cr}}^{(N)} \leq$ $c_{\mathrm{cr}}^{(N-1)} \leq \cdots \leq c_{\mathrm{cr}}^{(2)} \leq 1$ such that for sufficiently large $c>c_{\mathrm{cr}}^{(2)}$ only an asynchronous state is left. We investigate the main mechanisms generating this sequence of bifurcations analytically and give an intuitive explanation. We also discuss key consequences of this desynchronization mechanism for biophysically more detailed systems.

Consider a network of $N$ oscillatory neurons [8], whose state at time $t$ is characterized by a phase variable $\phi_{i}, i \in$ $\{1,2, \ldots, N\}$, that constantly increases with time $\frac{d}{d t} \phi_{i}=1$. The membrane potentials $u_{i}=U\left(\phi_{i}\right)$ are specified by a rise function $U$ that characterizes the subthreshold dynamics of a neuron. Here $U$ is smooth, strictly monotonically increasing $\left(U^{\prime}>0\right)$, and normalized to $U(0)=0$ and $U(1)=1$.

A neuron $j$ generates a spike when its membrane potential crosses a threshold, $u_{j}\left(t^{-}\right) \geq 1$, which in turn may trigger an avalanche of spikes: Neurons reaching the threshold due to the free time evolution define the triggering set $\Theta^{(0)}=\left\{j \mid u_{j}\left(t^{-}\right)=1\right\}$. The units $j \in \Theta^{(0)}$ generate spikes which are instantaneously received by all the neurons $i$ in the network. In response, their potentials are updated according to 


$$
u_{i}^{(1)}=u_{i}\left(t^{-}\right)+\sum_{j \in \Theta^{(0)}} \varepsilon_{i j},
$$

where $\varepsilon_{i j} \geq 0$ determines the strength of a directed synaptic connection from neuron $j$ to $i$. The initial pulse may trigger certain other neurons $k \in \Theta^{(1)}=\left\{k \mid u_{k}\left(t^{-}\right)<1 \leq\right.$ $\left.u_{k}^{(1)}\right\}$ to spike, etc. This process continues $n \leq N$ steps until no new neuron crosses the threshold. At each step $m \in$ $\{2,3, \ldots, n\}$ the potentials are updated according to

$$
u_{i}^{(m+1)}=u_{i}^{(m)}+\sum_{j \in \Theta^{(m)}} \varepsilon_{i j}
$$

where $\Theta^{(m)}=\left\{k \mid u_{k}^{(m-1)}<1 \leq u_{k}^{(m)}\right\}$. The phases immediately after the avalanche $\Theta=\bigcup_{m=0}^{n} \Theta^{(m)}$ of size $a=|\Theta|$ are obtained via

$$
\phi_{i}\left(t^{+}\right)= \begin{cases}U^{-1}\left(u_{i}\left(t^{-}\right)+\sum_{j \in \Theta} \varepsilon_{i j}\right) & i \notin \Theta \\ U^{-1}\left(R\left[u_{i}\left(t^{-}\right)+\sum_{j \in \Theta} \varepsilon_{i j}-1\right]\right) & i \in \Theta,\end{cases}
$$

where $R$ is the partial reset function. Here we focus on the linear form $R(\zeta)=c \zeta$, with $c \in[0,1]$ specifying the remaining fraction of suprathreshold input charges after reset. As a key example of the collective dynamics of neurons with partial reset, we here study neurons with convex rise function $\left(U^{\prime \prime}>0\right)$, modeling, e.g., a class of conductance based integrate-and-fire neurons, which are homogeneously and globally coupled without selfinteractions, $\varepsilon_{i j}=\left(1-\delta_{i j}\right) \tilde{\varepsilon}$, and total input strength $\varepsilon=$ $\sum_{j} \varepsilon_{i j}=(N-1) \tilde{\varepsilon}<1$. As explicit sample rise functions we use $U_{b}(\phi)=\frac{1}{b} \ln \left[1+\left(e^{b}-1\right) \phi\right]$, where $b<0$ specifies its curvature.

Systematic numerical investigations indicate a strong dependence of the network dynamics on the partial reset strength $c$ : In particular, we find synchronous states, cluster states, asynchronous states, and a sequential desynchronization of clusters with increasing $c$. More detailed, if $c$ is sufficiently small, the long-term dynamics is dominated by many coexisting cluster states in which neurons are synchronized to differently sized groups resulting in a periodic state of the network (cf. Fig. 1). As $c$ increases, less and less clusters are observed with the maximal number of units per cluster decreasing. Above a critical value of $c$ only an asynchronous state remains.

What is the origin of this rich repertoire of dynamics and which mechanisms control the observed transition? To answer these questions, we analytically investigate the existence and stability of periodic states with clusters of arbitrary sizes. It turns out that the sequence of bifurcations is controlled by two effects: subthreshold inputs that are always synchronizing and suprathreshold inputs that may be synchronizing or desynchronizing depending on the strength $c$ of the partial reset.

As the first step we show that the fully asynchronous (splay [9]) state exists and is stable for all $c \in[0,1]$. It is defined by identical interspike intervals between each pair
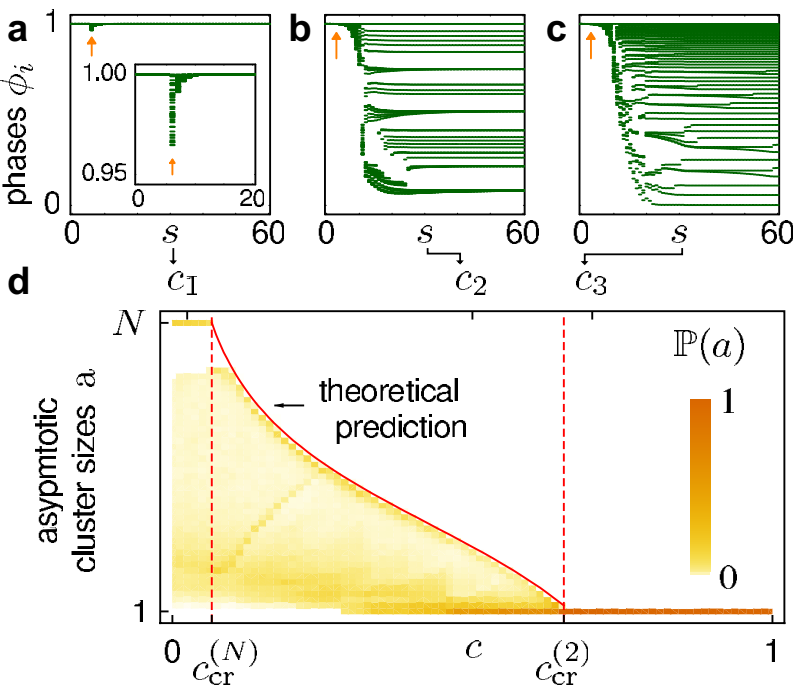

FIG. 1 (color online). Sequential desynchronization transition in a network of $N=50$ neurons $\left(U=U_{b}, b=-3, \tilde{\varepsilon}=\right.$ $0.0175)$. The phases $\phi_{i}$ of all neurons are plotted against the sth spike of a reference neuron. Starting from a synchronous state and perturbing at $s=5$, the phase dynamics are shown for (a) $c_{1}=0.025<c_{\mathrm{cr}}^{(N)}$ (inset: magnification), (b) $c_{2}=0.5 \in$ $\left(c_{\mathrm{cr}}^{(N)}, c_{\mathrm{cr}}^{(2)}\right)$, and (c) $c_{3}=0.7>c_{\mathrm{cr}}^{(2)}$. (d) Probability $\mathbb{P}(a)$ of observed cluster sizes $a$ in the asymptotic dynamics of 1500 simulations for each $c \in\{0,0.0125, \ldots, 1\}$ starting from random phases uniformly distributed in $[0,1)$. Solid red line: Exact theoretical prediction (10) above which clusters are unstable.

of subsequently and individually firing neurons. A firing map maps the phases $\phi_{i}$ of the system just before one avalanche to the phases just before the next. To construct this map for the asynchronous state we evaluate the current spike (a one-neuron "avalanche") and shift all phases by the same amount $\sigma$ such that the largest of the resulting phases is at threshold. Without loss of generality, we label the neurons' phases in ascending order such that the phases $\phi_{i}^{*}$ and the shift $\sigma^{*}$ uniquely define the asynchronous state; they are determined self-consistently by $\phi_{1}^{*}=\sigma^{*}>0$ and $\phi_{l}^{*}=U^{-1}\left[U\left(\phi_{l-1}^{*}\right)+\tilde{\varepsilon}\right]+\sigma^{*}$ for $l \in\{2, \ldots, N\}$ such that $\phi_{N}^{*}=1$. Homogeneity of the network implies invariance of such an asynchronous state under the firing map for every $\varepsilon<1$. Applying a small perturbation $\boldsymbol{\delta}^{(0)}=$ $\left(\delta_{1}^{(0)}, \ldots, \delta_{N-1}^{(0)}\right)$ to the $N-1$ phases which are not at threshold and linearizing the firing map (cf. $[10,11]$ ) yields the perturbations after the next firing

$$
\boldsymbol{\delta}^{(1)}=A \boldsymbol{\delta}^{(0)} .
$$

Here $A$ is a matrix whose only nonzero elements are $A_{i+1, i}=a_{i}$ for $i \in\{1, \ldots, N-2\}$ and $A_{i, N-1}=-a_{N-1}$, where

$$
a_{i}=\frac{U^{\prime}\left(\phi_{i}^{*}\right)}{U^{\prime}\left(U^{-1}\left[U\left(\phi_{i}^{*}\right)+\tilde{\varepsilon}\right]\right)}
$$

for $i \in\{1, \ldots, N-1\}$. Since $U^{\prime}>0, U^{\prime \prime}>0$, and $\tilde{\varepsilon}>0$, 
we have $a_{i}<1$. The Eneström-Kakeya theorem [12] applied to the characteristic polynomial of $A$ implies that its eigenvalues satisfy $\left|\lambda_{i}\right|<1$ for all $i \leq N-1$, showing that the asynchronous state is linearly stable. The stability properties of this state are identical for all $c \in[0,1]$ because all neurons fire individually and do not initiate any avalanche with suprathreshold inputs.

Next we investigate the stability properties of a periodic cluster state under the return map, i.e., the mapping of all phases just before the triggering of an avalanche $\Theta$ of size $a_{1}=|\Theta|$ to all phases just before the same avalanche reoccurs. Such a cluster state exists (i.e., is invariant) unless the maximal cluster size is too large such that this cluster absorbs neurons not belonging to it or is absorbed by other clusters. Given that the specific neuron $N \in \Theta^{(0)}$ stays in the triggering set of the avalanche, the return map $M$ equals the map defined from the hyperplane $\phi_{N}=1$ to itself. It is fully specified by the number $m$ of avalanches, $1 \leq m \leq N$, by the number $a_{s}, s \in\{1, \ldots, m\}$, of neurons spiking in each avalanche, and by the subsequent phase shifts $\sigma_{s}$ that fix the time lags between the avalanches. This information is determined from the initial phase vector $\left(\phi_{1}, \ldots, \phi_{N-1}, 1\right)$ and grouped into a firing sequence $\mathcal{F}=$ $\left[\left(\varepsilon_{s}, \sigma_{s}\right)\right]_{s=0}^{m}$, setting $\varepsilon_{s}=a_{s} \tilde{\varepsilon}$. For given $\mathcal{F}$ the return map then reads

$$
M_{\mathcal{F}}\left(\phi_{i}\right)=S_{\sigma_{m}} \circ H_{\varepsilon_{m}} \circ \cdots \circ S_{\sigma_{2}} \circ H_{\varepsilon_{2}} \circ S_{\sigma_{1}} \circ J_{\varepsilon_{1}}\left(\phi_{i}\right)
$$

for $i \in \Theta$. Here $S_{\sigma}(\phi)=\phi+\sigma$ mediates a pure phase shift, $H_{x}(\phi)=U^{-1}[U(\phi)+x]$ specifies the subthreshold response to an incoming spike, and $J_{x}(\phi)=$ $U^{-1}(R[U(\phi)+x-\tilde{\varepsilon}-1])$ represents the partial response $R$ to suprathreshold input. By definition we have $M_{\mathcal{F}}(1)=1$. For an avalanche of size $a_{1}$ to not decay into smaller subclusters, all neurons which were excited above threshold by $a<a_{1}$ preceding spikes in this avalanche [cf. (2)], i.e., with phases in the interval $I_{a}=\left[U^{-1}(1-a \tilde{\varepsilon}), 1\right]$, have to be excited above threshold again in the avalanche after return. Thus the conditions

$$
\mathcal{M}_{\mathcal{F}}\left(I_{a}\right) \subset I_{a}
$$

for all $a \in\left\{1, \ldots, a_{1}-1\right\}$ and all admissible firing sequences $\mathcal{F}$ with initial avalanche size $a_{1}$ ensure a cluster of size $a_{1}$ to not split up. For general $R$ and $U$ these conditions for most synchronizing and most desynchronizing firing sequences $\mathcal{F}$ yield upper and lower bounds on the maximal size of a cluster to be invariant under the return map [10]. Here we focus on the specific rise function $U_{b}(\phi)$ and on the linear partial reset $R(\zeta)=c \zeta$ to obtain explicit equations for the bifurcation points $c_{\mathrm{cr}}^{(a)}[(10)$ and (12) below]. For $U=U_{b}$ the change of phase differences due to subthreshold inputs is independent of the phase, i.e., $H_{\varepsilon}(\phi)-H_{\varepsilon}(\psi)=H_{\varepsilon} \circ S_{\sigma}(\phi)-H_{\varepsilon} \circ S_{\sigma}(\psi)$ for $\sigma \geq 0$. Using this property in (6) after determining $\sigma_{m}$ from $M_{\mathcal{F}}(1)=1$ the return map (6) for $i \in \Theta$ becomes independent of $\mathcal{F}$ and simplifies to

$$
M_{\mathcal{F}}\left(\phi_{i}\right)=S_{\bar{\sigma}} \circ H_{\left(N-a_{1}\right) \tilde{\varepsilon}} \circ J_{a_{1} \tilde{\varepsilon}}\left(\phi_{i}\right),
$$

where $\bar{\sigma}=1-H_{\left(N-a_{1}\right) \tilde{\varepsilon}} \circ J_{a_{1} \tilde{\varepsilon}}(1)$. Since $M_{\mathcal{F}}^{\prime} \geq 0$ conditions (7) simplify to $M_{\mathcal{F}}\left[U_{b}^{-1}(1-a \tilde{\varepsilon})\right] \geq U_{b}^{-1}(1-a \tilde{\varepsilon})$ for $a \in\left\{1, \ldots, a_{1}-1\right\}$. Using convexity $M_{\mathcal{F}}^{\prime \prime} \geq 0$ these further reduce to the single condition for $a=1$

$$
M_{\mathcal{F}}\left[U_{b}^{-1}(1-\tilde{\varepsilon})\right] \geq U_{b}^{-1}(1-\tilde{\varepsilon}),
$$

where a single unit triggers the avalanche. Conversely, a generic perturbation will disturb the cluster such that it gets triggered by a single unit only. Thus condition (9) is sufficient and necessary for avalanches of size $a_{1}$ to be invariant under the return map. Using equality in (9), the bifurcation values $c_{\mathrm{cr}}^{(a)}$ above which a cluster state with maximal cluster size $a$ becomes unstable are then determined implicitly by the equation

$$
e^{b\left(1-\left[(N-a)+c_{\mathrm{cr}}^{(a)}(a-1)\right] \tilde{\varepsilon}\right)}\left(e^{-b \tilde{\varepsilon}}-1\right)=\left(e^{-b c_{\mathrm{cr}}^{(a)} \tilde{\varepsilon}}-1\right) .
$$

Figure 1 shows an explicit example of these theoretical predictions for the bifurcation values $c_{\mathrm{cr}}^{(a)}$ which well match the numerical results.

In general, we infer from (10) that

$$
0<c_{\mathrm{cr}}^{(N)}<c_{\mathrm{cr}}^{(N-1)}<\cdots<c_{\mathrm{cr}}^{(2)}<1,
$$

independent of specific parameters $b, \tilde{\varepsilon}$, and $N$. First, this implies that the entire sequence of bifurcations is guaranteed to occur in the physically relevant open interval $c \in$ $(0,1)$. Second, with increasing $c$, states with larger clusters become unstable before states with smaller clusters. In particular, the synchronous state becomes unstable first and cluster states with at most two synchronized neurons become unstable last. Third, for $a=2$ we find that the largest bifurcation point

$$
c_{\mathrm{cr}}^{(2)}=\frac{1}{b \tilde{\varepsilon}} \ln \left[1-e^{b[1-(N-1) \tilde{\varepsilon}]}\left(1-e^{b \tilde{\varepsilon}}\right)\right]
$$

can be arbitrarily small, e.g., as $b \rightarrow-\infty$. Thus the entire sequence of desynchronizing bifurcations can occur for arbitrary small $c$.

The form (8) of the return map reveals the mechanisms underlying the desynchronization transition as the interplay between synchronization due to subthreshold inputs mediated by $H_{(N-a) \tilde{\varepsilon}}$ [cf. Fig. 2(a)] and further synchronization or desynchronization due to suprathreshold inputs and partial reset mediated by $J_{a \tilde{\varepsilon}}$, depending on the strength of the partial reset [cf. Figs. 2(b) and 2(c)]. The large clusters get unstable first since they receive less synchronizing subthreshold inputs from the other smaller clusters and additionally the desynchronization due to the reset is stronger in larger avalanches.

The observed desynchronization transition prevails for networks of inhomogeneously coupled units and neurons with rise functions of mixed convex and concave curvature, as, e.g., characteristic for quadratic integrate-and-fire neurons [13], the normal form of type I excitable neurons. 

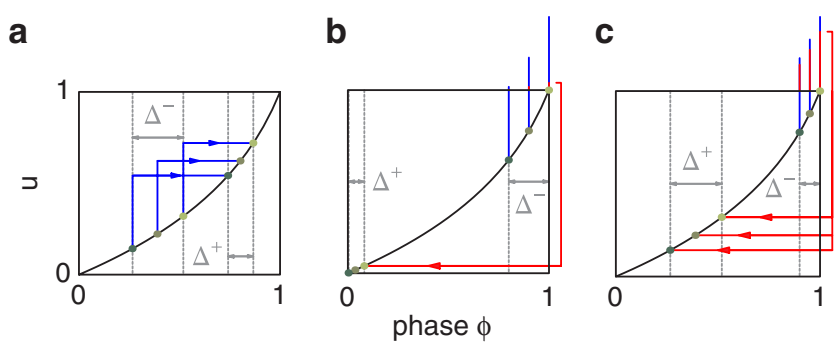

FIG. 2 (color online). Coacting synchronizing and desynchronizing mechanisms underly the desynchronization transition. The phase-potential relations are shown as solid black curves. The phase differences before $\left(\Delta^{-}\right)$and after spike reception $\left(\Delta^{+}\right)$are indicated. (a) Synchronization due to subthreshold inputs coacting with either (b) synchronization for strongly refractory partial resets $(c \approx 0)$ or $(\mathrm{c})$ desynchronization for sufficiently conservative partial resets $(c \approx 1)$ determine the invariance of clusters under the return map (6).

Moreover, our simple model system can be connected to biophysically more detailed type I models by comparing spike time response curves that encode the shortening of the interspike intervals (ISI) following an excitatory input at different phases of the neural oscillation. An excitatory stimulus that causes the neuron to spike will maximally shorten the ISI in which the stimulus is applied. Additionally the following ISI is typically affected as well. This effect can be characterized by an appropriately chosen partial reset in our simple system [10]. Networks of two-compartment conductance based neurons indeed exhibit similar desynchronization transitions when varying the coupling between soma and dendrite (not shown) which in our simplified model controls the partial reset.

In summary, we introduced a simple model of spiking neurons with partial reset to investigate collective network effects of possible residual charges that may be important after somatic reset. Already for globally and homogeneously coupled networks we find that residual charges present after spike generation drastically affect the network dynamics. We revealed a new desynchronization mechanism that controls a sequential destabilization of cluster states. For no or only small fractions of conserved charge, $c \in\left[0, c_{\mathrm{cr}}^{(N)}\right)$, the synchronous state and cluster states with many different cluster sizes coexist, whereas for large fractions, $c \in\left(c_{\mathrm{cr}}^{(2)}, 1\right]$, only the asynchronous state is left. For intermediate $c \in\left[c_{\mathrm{cr}}^{(N)}, c_{\mathrm{cr}}^{(2)}\right]$ there is a sequence of bifurcations, each destabilizing the largest stable cluster. Interestingly, this entire sequence may occur in an arbitrarily small interval near $c=0$.

The mechanism for neural desynchronization discussed above differs strongly from known mechanisms that are based, e.g., on heterogeneity, noise, or delayed feedback $[14,15]$. Possibly, the mechanism uncovered here may also be used in modified form to prevent synchronization in neural systems like in Parkinson tremor or in epileptic seizures [15]. This calls for a future systematic study of the impact of $c$ and related parameters that modulate local response properties and thus synchronization [16]. The simple model system introduced above offers the first example of an analytically tractable network model which, based on partial reset, characterizes an essential feature of biophysically detailed compartmental models [5,17].

M. T. acknowledges support by the Federal Ministry of Education and Research (BMBF), Germany, under Grant No. 01GQ0430. C. K. acknowledges financial support by the German Academic Exchange Service (DAAD).

[1] C. Koch, Biophysics of Computation (Oxford University, New York, 1999); L. F. Abbott and P. Dayan, Theoretical Neuroscience (MIT , Cambridge, MA, 2001).

[2] I. Segev, J. W. Fleshman, and R. E. Bruke, in Methods in Neuronal Modeling, edited by C. Koch and I. Segev (MIT, Cambridge, MA, 1998).

[3] P. C. Bressloff, Physica (Amsterdam) 80D, 399 (1995).

[4] J. P. Rospars and P. Lansky, Biol. Cybern. 69, 283 (1993).

[5] Z.F. Mainen and T. J. Sejnowski, Nature (London) 382, 363 (1996).

[6] J. J. Hopfield and A. V. M. Herz, Proc. Natl. Acad. Sci. U.S.A. 92, 6655 (1995); P. C. Bressloff, S. Coombes, and B. de Souza, Phys. Rev. Lett. 79, 2791 (1997); P.C. Bressloff, Neural Comput. 12, 91 (2000); W. Gerstner and J. L. van Hemmen, Phys. Rev. Lett. 71, 312 (1993).

[7] A. Pikovsky, O. Popovych, and Yu. Maistrenko, Phys. Rev. Lett. 87, 044102 (2001); K. Kaneko, Phys. Rev. Lett. 78, 2736 (1997).

[8] R. E. Mirollo and S. H. Strogatz, SIAM J. Appl. Math. 50, 1645 (1990); U. Ernst, K. Pawelzik, and T. Geisel, Phys. Rev. Lett. 74, 1570 (1995).

[9] S. H. Strogatz and R. E. Mirollo, Phys. Rev. E 47, 220 (1993); R. Zillmer, R. Livi, A. Politi, and A. Torcini, Phys. Rev. E 76, 046102 (2007).

[10] C. Kirst and M. Timme, arXiv:0812.1786v1.

[11] M. Timme, F. Wolf, and T. Geisel, Phys. Rev. Lett. 92, 074101 (2004); S. Jahnke, R. M. Memmesheimer, and M. Timme, Phys. Rev. Lett. 100, 048102 (2008).

[12] For all zeros $\lambda$ of $p_{n}(z)=\sum_{i=0}^{n} c_{i} z^{i}, c_{i}>0, c_{n+1}=1$, $|\lambda| \leq \max _{0 \leq i<n}\left\{c_{i} / c_{i+1}\right\}$. R. A. Horn and C. R. Johnson, Matrix Analysis (Cambridge University Press, Cambridge, England, 1996).

[13] B. Ermentrout, Neural Comput. 8, 979 (1996); D. Hansel and G. Mato, Phys. Rev. Lett. 86, 4175 (2001).

[14] C. van Vreeswijk, L.F. Abbott, and G. B. Ermentrout, J. Comput. Neurosci. 1, 313 (1994); C. van Vreeswijk, Phys. Rev. Lett. 84, 5110 (2000).

[15] Y. Maistrenko, O. Popovych, O. Burylko, and P. A. Tass, Phys. Rev. Lett. 93, 084102 (2004); I. Z. Kiss et al., Science 316, 1886 (2007); O. V. Popovych, C. Hauptmann, and P. A. Tass, Phys. Rev. Lett. 94, 164102 (2005); O. E. Omel'chenko, Y. Maistrenko, and P. A. Tass, Phys. Rev. Lett. 100, 044105 (2008).

[16] C. Kirst and M. Timme, Phys. Rev. E 78, 065201(R) (2008).

[17] A. M. Oswald, B. Dorion, and L. Maler, J. Neurophysiol. 97, 2731 (2007). 